\title{
Pineal cysts and other pineal region malignancies: determining factors predictive of hydrocephalus and malignancy
}

\author{
*Robert M. Starke, MD, MSc, ${ }^{1}$ Justin M. Cappuzzo, MD, ${ }^{2}$ Nicholas J. Erickson, BS, ${ }^{2}$ and \\ Jonathan H. Sherman, MD³ \\ 1Department of Neurosurgery, University of Virginia, Charlottesville, Virginia; ${ }^{2}$ School of Medicine and Health Sciences; and \\ ${ }^{3}$ Department of Neurological Surgery, The George Washington University, Washington, DC
}

\begin{abstract}
OBJECTIVE Cystic lesions of the pineal gland are most often uncomplicated benign lesions with typical MRI characteristics. The authors aimed to study pineal lesion characteristics on MRI to better distinguish benign pineal cysts from other pineal region malignancies as well as to determine which characteristics were predictive of the latter malignancies. They also aimed to study risk factors predictive of hydrocephalus or malignancy in patients harboring these lesions.

METHODS The authors performed a retrospective review of a prospectively compiled database documenting the outcomes of patients with suspected pineal cysts on MRI who had presented in the period from 1998 to 2004. Inherent patient and lesion characteristics were assessed in a univariate logistic regression analysis to predict the following dependent variables: development of hydrocephalus, biopsy-confirmed malignancy, and intervention. Possible inherent patient and lesion characteristics included age, sex, T1 and T2 MRI signal pattern, contrast enhancement pattern, presence of cyst, presence of blood, complexity of lesion, presence of calcification, and duration of follow-up. Inherent patient and lesion characteristics that were predictive in the univariate analysis $(p<0.15)$ were included in the multivariable logistic regression analysis.
\end{abstract}

RESULTS Of the 79 patients with benign-appearing pineal cysts, $26(33 \%)$ were male and $53(67 \%)$ were female, with a median age of 38 years (range 9-86 years). The median cyst radius was $5 \mathrm{~mm}$ (range 1-20 mm). Two patients $(2.5 \%)$ had evidence of calcifications, 7 (9\%) had multicystic lesions, and 25 (32\%) had some evidence of contrast enhancement.

The median follow-up interval was 3 years (range $0.5-13$ years). Seven patients (9\%) had an increase in the size of their lesion over time. Eight patients (10\%) had a hemorrhage, and 11 patients (14\%) developed hydrocephalus. Nine (11\%) received ventriculoperitoneal shunts for the development of hydrocephalus, and 12 patients $(16 \%)$ were found to have malignancies following biopsy or resection. In the multivariate analysis, contrast enhancement on MRI (OR 1.6, 95\% $\mathrm{Cl} 2.86-74.74, p=0.013)$ and hemorrhage $(\mathrm{OR} 26.9,95 \% \mathrm{Cl} 3.4-212.7, p=0.022)$ were predictive of hydrocephalus. Increasing lesion size and hydrocephalus were near perfect predictors of malignancy and thus were removed from multivariate analysis. In addition, contrast enhancement on MRI (OR 8.8, 95\% $\mathrm{Cl} 2.0-38.6, p=0.004)$ and hemorrhage (OR 6.8, 95\% Cl 1.1-40.5, $p=0.036$ ) were predictive of malignancy.

CONCLUSIONS Although cystic abnormalities of the pineal gland are often benign lesions, they are frequently monitored over time, as other pineal region pathologies may appear similarly on MRI. Patients with growing lesions, contrast enhancement, and hemorrhage on MRI are more likely to develop hydrocephalus and have malignant pathology on histological examination and should therefore be followed up with serial MRI with a lower threshold for neurosurgical intervention.

https://thejns.org/doi/abs/10.3171/2016.8.JNS16220

KEY WORDS pineal cyst; hemorrhage; adult; aqueductal stenosis; hydrocephalus

ABBREVIATIONS EVD = external ventricular drainage.

SUBMITTED January 30, 2016. ACCEPTED August 1, 2016.

INCLUDE WHEN CITING Published online October 21, 2016; DOI: 10.3171/2016.8.JNS16220.

* Drs. Starke and Cappuzzo contributed equally to this work. 
$\mathrm{P}$ INEAL cysts are commonly occurring lesions, with a reported incidence in roughly $1 \%-4 \%$ of individuals undergoing standard MRI studies. . $^{1,6,10,14,16,19,20,25,42}$ Autopsy series have shown the rate to be even greater, with occurrence in $25 \%-40 \%$ of subjects. ${ }^{4,11,18,34,37}$ Their incidence is typically higher in women, with lesions most frequently occurring in the 2 nd decade of life and subsequently decreasing in frequency with age. ${ }^{39}$ These cysts are traditionally benign and are normal variants of the pineal gland composed of cystic structures surrounded by parenchyma. $^{\text {? }}$

Patients with pineal cysts are typically asymptomatic; however, they can also present with a broad array of symptoms, with headache being the most frequent., ${ }^{7,86}$ Interestingly, these headaches were once thought to be caused by increased intracranial pressure, but new research suggests that a hormonal imbalance involving melatonin may be to blame. . $12,24,27,35,36,40,45$ Other commonly encountered symptoms include seizures, vertigo, blurred vision, hemiparesis, and emesis. $4,14,26,43,46$

The radiological appearance of pineal cysts varies based on the imaging modality used. Magnetic resonance imaging with gadolinium contrast enhancement is the preferred radiological method, allowing for greater evaluation of lesion size, vascularity, composition, and anatomical relationships. ${ }^{7,32,47}$ Despite all of the advances in high-resolution MRI, however, there is no current definitive method by which we can distinguish benign pineal cysts from pineal region malignancies such as pineocytomas, pineoblastomas, germinomas, or mature teratomas, particularly when any of these pathologies can often include cystic components visible on imaging. ${ }^{11}$ Therefore, we assessed the imaging characteristics of pineal cystic lesions with the goal of differentiating between asymptomatic benign pineal cysts and pineal malignancies. We additionally aimed to identify any characteristics of these cysts that would predict the development of hydrocephalus.

\section{Methods}

We performed a retrospective review of patients reported to have pineal cysts on MRI of the brain at the University of Virginia between 1998 and 2004. These patients had all been referred to the neurosurgery service as outpatients and were followed up over time with serial MRI. For each patient we subsequently collected and documented the appropriate demographics and clinical details, including age, sex, duration of follow-up, indications for initial imaging, histology of malignancy if present, and clinical outcome.

Additionally, we collected and documented the imaging characteristics for each patient, including lesion diameter, T1 or T2 imaging pattern (hyperintensity, hypointensity, or isointensity), postcontrast enhancement pattern, presence of calcifications, any multiplicity of cysts, whether hemorrhage was present (as evidenced by hyperdensity on head CT or hyperintensity on brain MRI), and whether there was any increase in the size of the lesion over time. Finally, we identified subsets of patients who had presented with hydrocephalus and underwent procedural intervention (including external ventricular drainage [EVD], shunt placement, or resection), as well as patients who underwent resection and/or biopsy and had a histological diagnosis of pineal malignancy.

We reviewed our findings to identify any apparent difference in imaging characteristics between benign cysts and malignancies and between symptomatic and asymptomatic benign cysts. For purposes of analysis, cysts were considered "simple" if their imaging characteristics did not contain any of the following: a multicystic component, a hemorrhagic component, calcification, or postcontrast enhancement.

\section{Statistical Analysis}

Data are presented as the mean and range for continuous variables and as the frequency for categorical variables. Calculations of normality were performed using the ladder of powers. Statistical analyses of categorical variables were performed using the chi-square and Fisher's exact tests, as appropriate. Statistics of means were performed using an unpaired Student t-test both with and without equal variance (Levene's test), as necessary, and Wilcoxon rank-sum tests when variables were not normally distributed. Analysis of variance followed by Bonferroni post hoc testing was used to assess means between 3 or more groups. Odds ratios and 95\% confidence intervals were calculated using univariate logistic regression analysis. Inherent patient and lesion characteristics were assessed as independent variables to predict the following dependent variables: biopsy-confirmed malignancy, development of hydrocephalus, and intervention (EVD, shunt, resection).

Possible inherent patient and lesion characteristics included age, sex, T1 and T2 MRI signal pattern, contrast enhancement pattern, presence of cyst, presence of blood, complexity of lesion, presence of calcification, and duration of follow up.

Inherent patient and lesion characteristics predicting biopsy-confirmed malignancy with a univariate $\mathrm{p}<$ 0.15 were included in a multivariable logistic regression analysis to assess the most robust predictors of having a biopsy-proven malignancy. Inherent lesion characteristics predicting the development of hydrocephalus with a univariate $p<0.15$ were included in a multivariable logistic regression analysis to assess the most robust predictors of the development of hydrocephalus. Inherent lesion characteristics predicting the necessity of a procedure (EVD, shunt, resection) with a univariate $\mathrm{p}<0.15$ were included in a multivariable logistic regression analysis to assess the most robust predictors of the need for an intervention. A p value $\leq 0.05$ was considered statistically significant.

\section{Results}

Of the 79 patients with benign-appearing pineal cysts, $26(33 \%)$ were male and $53(67 \%)$ were female, with a median age of 38 years (range 9-86 years; Table 1). The median cyst radius was $5 \mathrm{~mm}$ (range $1-20 \mathrm{~mm}$ ). Two patients (2.5\%) had evidence of calcifications, 7 (9\%) had multicystic lesions, and $25(32 \%)$ had some evidence of contrast enhancement. Twelve patients (15\%) had cysts displaying a peripheral enhancement pattern, whereas $13(16 \%)$ had another type of contrast enhancement. 
TABLE 1. Summary of patient characteristics

\begin{tabular}{lc}
\hline \multicolumn{1}{c}{ Characteristic } & Value \\
\hline No. of patients & 79 \\
\hline Median age at diagnosis in yrs (range) & $38(9-86)$ \\
\hline Sex & $26(33 \%)$ \\
\hline Male & $53(67 \%)$ \\
\hline Female & $2(2.5 \%)$ \\
\hline Calcifications & $7(9 \%)$ \\
\hline Multicystic & $25(32 \%)$ \\
\hline Contrast enhancement & $12(15 \%)$ \\
\hline Enhancement pattern & $13(16 \%)$ \\
\hline Peripheral & $3(0.5-13)$ \\
\hline Nonperipheral & $7(9 \%)$ \\
\hline Median FU in yrs (range) & $8(10 \%)$ \\
\hline Interval increase in lesion size & $11(14 \%)$ \\
\hline Hemorrhage & $11(14 \%)$ \\
\hline Hydrocephalus & \\
\hline No. of patients undergoing procedure & $3(4 \%)$ \\
\hline Type of procedure & $9(11 \%)$ \\
\hline EVD & $6(8 \%)$ \\
\hline VPS & $12(16 \%)$ \\
\hline Resection & \\
\hline Malignancy & \\
\hline
\end{tabular}

$\mathrm{FU}=$ follow-up; VPS = ventriculoperitoneal shunting.

The median follow-up interval was 3 years (range 0.513 years). Seven patients (9\%) had an increase in the size of their lesion over time. Eight patients (10\%) had a hemorrhage, and 11 patients (14\%) developed hydrocephalus. Nine (11\%) received ventriculoperitoneal shunts, and 12 patients $(16 \%)$ were found to have malignant histology following biopsy or resection. In a multivariate analysis, contrast enhancement on MRI (OR 1.6, p = 0.013) and hemorrhage (OR 26.9, 95\% CI 3.4-212.7, $\mathrm{p}=0.022$ ) were predictive of hydrocephalus (Table 2). Increasing lesion size and hydrocephalus were near perfect predictors of pineal region malignancy and thus were removed from multivariate analysis. In addition, contrast enhancement on MRI (OR 8.8, 95\% CI 2.0-38.6, $\mathrm{p}=0.004$ ) and hemorrhage (OR 6.8, 95\% CI 1.1-40.5, $\mathrm{p}=0.036)$ were predictive of a pineal malignancy (Table 3).

\section{Discussion}

As the use of MRI has become more frequent, the number of incidentally discovered pineal cysts has greatly increased. ${ }^{1,6,10,14,16,19,20,25,42}$ Despite the advances made with respect to high-resolution MRI, however, there is no clearly defined means of distinguishing these cysts from other pineal region malignancies. ${ }^{11}$ It is generally accepted that typical benign pineal cysts are small $(<1 \mathrm{~cm}$ in diameter) and have a round to oval appearance with smooth boundaries and a cyst wall with a maximum thickness of $2 \mathrm{~mm}$ on radiological studies. ${ }^{9,15}$ In our patient population, we found a median cyst radius of $5 \mathrm{~mm}$ (range 1-20 mm). The average radius of pineal lesions ultimately diagnosed as pineal malignancy was $12.3 \mathrm{~mm}$, whereas the average radius of simple cysts was $5.4 \mathrm{~mm}$ and the average radius of lesions requiring a procedure (EVD, shunt, resection) was $9.7 \mathrm{~mm}$. This need for intervention is most likely due to the increasing degree of compression of the rostral third ventricle with an enlarging lesion, causing hydrocephalus or general mass effect, with the subsequent production of neurological symptoms. We also found that smaller lesions are more frequently benign pineal cysts.

The etiology of pineal cysts is still under debate; however, several postulates have been presented in an attempt to explain their origin. The first theory is that pineal cysts could be the congenital remnant of the embryological diverticulum that develops from the third ventricle and forms the pineal gland. ${ }^{18,20,25}$ The second theory is that these cysts could be an embryogenic remnant of the cavum pineale. ${ }^{9}$ Additional theories suggest that they may be the result of the degeneration of pinealocytes ${ }^{13,22}$ or a byproduct of ischemic necrosis. ${ }^{23}$ The most current theories, however, suggest formation via hemorrhage $e^{1,13,15,22,28}$ or a hormonal cause. ${ }^{8}$ As stated previously, the incidence of pineal cysts is typically higher in women, with lesions most frequently occurring in the 2nd decade of life and subsequently decreasing in occurrence with age..$^{39}$ Many authors have acknowledged a female predominance specifically consisting of a subgroup in the reproductive age range (age 15-49 years). Our study confirmed this predominance, which may support a hormonal cause for pineal cyst formation; however, further in-depth analysis is necessary.

On a similar note, hormonal imbalance may also be responsible for the headache that many pineal cyst patients present with. As noted previously, patients with

TABLE 2. Statistical analysis: factors predictive of hydrocephalus

\begin{tabular}{|c|c|c|c|c|c|c|}
\hline \multirow[b]{2}{*}{ Variable } & \multicolumn{3}{|c|}{ Univariate Analysis } & \multicolumn{3}{|c|}{ Multivariate Analysis } \\
\hline & OR & $95 \% \mathrm{Cl}$ & $p$ Value & OR & $95 \% \mathrm{Cl}$ & $p$ Value \\
\hline Age & 1.00 & $0.9-1.0$ & 0.913 & - & - & - \\
\hline Sex & 1.2 & $0.3-4.5$ & 0.793 & - & - & - \\
\hline FU & 1.0 & $1.0-1.1$ & 0.393 & - & - & - \\
\hline Hemorrhage & 37.2 & $6.3-249.5$ & $<0.001$ & 26.9 & $3.4-212.7$ & 0.022 \\
\hline Multicystic & 6.0 & $1.3-31.8$ & 0.035 & - & - & - \\
\hline Contrast enhancement & 14.6 & $2.9-74.8$ & 0.01 & 1.60 & $2.86-74.74$ & 0.013 \\
\hline T1/T2 pattern* & 6.1 & $2.2-17.1$ & - & - & - & - \\
\hline
\end{tabular}

* T1/T2 MRI signaling pattern. 
TABLE 3. Statistical analysis: factors predictive of pineal malignancy

\begin{tabular}{|c|c|c|c|c|c|c|}
\hline \multirow[b]{2}{*}{ Variable } & \multicolumn{3}{|c|}{ Univariate Analysis } & \multicolumn{3}{|c|}{ Multivariate Analysis } \\
\hline & OR & $95 \% \mathrm{Cl}$ & p Value & OR & $95 \% \mathrm{Cl}$ & $p$ Value \\
\hline Age & 1.0 & $0.9-1.0$ & 0.343 & - & - & - \\
\hline Sex & 2.3 & $0.7-8.2$ & 0.189 & - & - & - \\
\hline FU & 1.00 & $0.9-1.0$ & 0.904 & - & - & - \\
\hline Hemorrhage & 14.29 & $2.8-73.0$ & 0.001 & 6.8 & $1.1-40.5$ & 0.036 \\
\hline Multicystic & 6.67 & $1.2-38.2$ & 0.033 & - & - & - \\
\hline Contrast enhancement & 12.75 & $3.0-54.4$ & 0.001 & 8.8 & $2.0-38.6$ & 0.004 \\
\hline T1/T2 pattern & 7.50 & $2.35-23.88$ & 0.001 & - & - & - \\
\hline
\end{tabular}

pineal cysts are typically asymptomatic; however, headache is the most common presenting symptom among a broad list of possible symptoms. ${ }^{7,836}$ The cause of these headaches was initially thought to be increased intracranial pressure caused by hydrocephalus due to superior compression of the third ventricle by larger lesions. Larger lesions can cause compression of adjacent structures, including rostral compression of the third ventricle, leading to hydrocephalus. ${ }^{7}$ Unfortunately, given the location of the pineal gland, even smaller lesions can cause symptoms. ${ }^{8}$ Direct compression of the midbrain can result in motor and sensory disturbances. ${ }^{22}$ Other commonly encountered symptoms include seizures, vertigo, blurred vision, hemiparesis, and emesis. ${ }^{4,14,26,43,46}$ Pineal apoplexy (caused by hemorrhage), Parinaud's phenomenon (caused by compression of the superior colliculus), parkinsonian symptoms, choroid plexus papilloma formation, aseptic meningitis (due to cyst rupture), pseudo-precocious puberty (due to immaturity of the hypothalamic-gonadal axis), and diabetes insipidus are less frequent yet reported symptoms. ${ }^{21,26,30,31,37,38,41}$ There is also thought to be a hormonal and melatonin imbalance in most of these patients with headaches. In our patient population, we found that headache was one of the most common indications for undergoing MRI, with a total of 16 patients $(20 \%)$ presenting with this symptom. Moreover, of the 16 patients with headaches, $9(56 \%)$ of them were reproductive-age females, suggesting that there may indeed be a hormonal cause behind these headaches.

Pineal lesions usually lack nodularity or heterogeneous enhancement and are stable over time. ${ }^{3}$ Their radiological appearance varies based on the imaging modality used. Magnetic resonance imaging with gadolinium contrast enhancement is the preferred radiological method, allowing for greater evaluation of lesion size, vascularity, composition, and anatomical relationships. ${ }^{7,32,47}$ Occasionally, however, CT may be used, particularly for the initial scan before the diagnosis of a pineal cyst is made. These cysts can be either hypodense or undetectable on $\mathrm{CT}$ as the fluid may be similar in density to CSF. ${ }^{8,12,13,44}$ Hyperdensity may indicate hemorrhage or calcification. ${ }^{28,33}$ On MRI, pineal cysts appear hypointense to white matter and either isointense or diffusely hyperintense to CSF on T1-weighted sequences. This appearance on MRI is variable based on the protein content of the cystic fluid, prior hemorrhage, or stagnation of the fluid. ${ }^{8}$ With the addition of contrast, there is typically thin rim enhancement given the lack of a blood-brain barrier. ${ }^{8}$ On T2-weighted imaging, there is usually a homogeneous appearance that is isointense or slightly hyperintense relative to the CSF, which is thought to be mainly secondary to stagnation of the cystic fluid. ${ }^{8}$ Proton density and FLAIR sequences appear isointense or slightly hyperintense and slightly hyperintense in comparison to CSF, respectively. ${ }^{8}$

We found that patients with a complex pattern on MRI, as well as those with postcontrast lesion enhancement, were much more likely to have malignant lesions; $13(52 \%)$ of 25 patients with a complex imaging structure on MRI ultimately had a lesion diagnosed as a malignancy. Pineal cysts can also have rim enhancement after contrast administration. The type of enhancement varied in our study, and a large percentage (70\%) of lesions with nonperipheral enhancement were ultimately diagnosed as malignancies.

In a multivariate analysis, we found the most accurate predictor of malignancy to be cyst enhancement on MRI (OR 8.8, 95\% CI 2.0-38.6, $\mathrm{p}=0.004$ ) and hemorrhage (OR 6.8, 95\% CI 1.1-40.5, $\mathrm{p}=0.036$ ). Increasing lesion size and hydrocephalus were near perfect predictors of malignancy and thus were removed from multivariate analysis.

Similarly, we found on multivariate analysis that contrast enhancement of cysts on MRI (OR 1.6, p = 0.013) and hemorrhage (OR 26.9, 95\% CI 3.4-212.7, $\mathrm{p}=0.002$ ) were predictive of hydrocephalus. A complex imaging pattern was a near-perfect predictor and thus was removed from the multivariate analysis.

We believe that this information will provide possible insight into determining which patients with pineal cysts should be monitored over time. There is still much contradiction in the literature about the most appropriate therapeutic approach to pineal cysts as the natural history remains unclear, particularly with regard to asymptomatic pineal cysts. The management of asymptomatic cysts ranges from the absence of any follow-up to possible surgical intervention; however, the most common practice is to refrain from surgical intervention in an asymptomatic patient. Additionally, while some clinicians do not support routine imaging in adults with known pineal cysts, others recommend annual follow-up with clinical examination and imaging. ${ }^{2,17}$ Routine clinical examination coupled with routine imaging is highly recommended in any child with a pineal cyst. ${ }^{1,26,29,33,48}$ In symptomatic patients, particularly those with hydrocephalus, treatment can be divided 
into shunt placement, cyst excision, endoscopic or stereotactic aspiration, and endoscopic third ventriculostomy. ${ }^{5,17}$

As stated, there has been no uniform management of patients with pineal cysts found on MRI, and the question of which patients to follow up over time has remained largely unanswered. Given our findings, we recommend that all patients with pineal region lesions that enlarge over time, have contrast enhancement, or have hemorrhage on MRI should undergo routine follow-up, as their lesions have a much greater likelihood of ultimately being diagnosed as a malignancy. Further investigation is needed to determine whether the rest of these patients without the above characteristics necessitate follow-up over time.

This study is not without limitations. The small sample size and retrospective nature are inherent weaknesses to the overall design of the study. Additionally, the population studied (patients referred to the outpatient neurosurgery clinic) skews the sample such that there are a greater number of pineal region malignancies that were eventually diagnosed within this group. Furthermore, as this study focuses on patients referred to the neurosurgery outpatient clinic between 1998 and 2004, imaging and other records are no longer available. Nonetheless, these study limitations do not detract from the main purpose of the paper, which was to distinguish imaging and lesion characteristics that would be predictive of hydrocephalus and tumor and offer a means by which benign pineal cysts and pineal region tumors could be distinguished from one another on MRI.

\section{Conclusions}

Benign pineal cysts are a common occurrence and can be difficult to distinguish from other pathologies of the pineal region. Furthermore, no specific management guidelines exist regarding follow-up of these lesions and whether patients need to be monitored over time. Although a majority of patients will not have pineal malignancies, those who present with growing lesions, contrast enhancement, and hemorrhage are more likely to develop hydrocephalus and to have malignant pathology on histological examination and should therefore be followed up with serial MRI with a lower threshold for neurosurgical intervention.

\section{Acknowledgments}

John A. Jane Sr., MD, PhD (1931-2015), Department of Neurosurgery, University of Virginia, contributed to this manuscript by collaborating with the authors in developing this research topic.

\section{References}

1. Al-Holou WN, Garton HJ, Muraszko KM, Ibrahim M, Maher CO: Prevalence of pineal cysts in children and young adults. Clinical article. J Neurosurg Pediatr 4:230-236, 2009

2. Al-Holou WN, Terman SW, Kilburg C, Garton HJ, Muraszko KM, Chandler WF, et al: Prevalence and natural history of pineal cysts in adults. J Neurosurg 115:1106-1114, 2011

3. Apuzzo ML, Davey LM, Manuelidis EE: Pineal apoplexy associated with anticoagulant therapy. Case report. J Neurosurg 45:223-226, 1976

4. Barboriak DP, Lee L, Provenzale JM: Serial MR imaging of pineal cysts: implications for natural history and follow-up. AJR Am J Roentgenol 176:737-743, 2001

5. Berhouma M, Ni H, Delabar V, Tahhan N, Memou Salem $\mathrm{S}$, Mottolese C, et al: Update on the management of pineal cysts: Case series and a review of the literature. Neurochirurgie 61:201-207, 2015

6. Bodensteiner JB, Schaefer GB, Keller GM, McConnell JR: Incidental pineal cysts in a prospectively ascertained normal cohort. Clin Pediatr (Phila) 35:277-279, 1996

7. Bruce JN: Pineal tumors, in Winn HR (ed): Youmans Neurological Surgery, ed 6. Philadelphia: Saunders Elsevier, 2011, pp 1359-1372

8. Choy W, Kim W, Spasic M, Voth B, Yew A, Yang I: Pineal cyst: a review of clinical and radiological features. Neurosurg Clin N Am 22:341-351, vii, 2011

9. Cooper ER: The human pineal gland and pineal cysts. J Anat 67:28-46, 1932

10. Di Costanzo A, Tedeschi G, Di Salle F, Golia F, Morrone R, Bonavita V: Pineal cysts: an incidental MRI finding? J Neurol Neurosurg Psychiatry 56:207-208, 1993

11. Engel U, Gottschalk S, Niehaus L, Lehmann R, May C, Vogel S, et al: Cystic lesions of the pineal region-MRI and pathology. Neuroradiology 42:399-402, 2000

12. Evans RW, Peres MF: Headaches and pineal cysts. Headache 50:666-668, 2010

13. Fain JS, Tomlinson FH, Scheithauer BW, Parisi JE, Fletcher GP, Kelly PJ, et al: Symptomatic glial cysts of the pineal gland. J Neurosurg 80:454-460, 1994

14. Fetell MR, Bruce JN, Burke AM, Cross DT, Torres RA, Powers JM, et al: Non-neoplastic pineal cysts. Neurology 41:1034-1040, 1991

15. Fleege MA, Miller GM, Fletcher GP, Fain JS, Scheithauer BW: Benign glial cysts of the pineal gland: unusual imaging characteristics with histologic correlation. AJNR Am J Neuroradiol 15:161-166, 1994

16. Golzarian J, Balériaux D, Bank WO, Matos C, Flament-Durand J: Pineal cyst: normal or pathological? Neuroradiology 35:251-253, 1993

17. Greenberg MS: Pineal cysts, in Handbook of Neurosurgery. New York: Thieme, 2010, pp 691-692

18. Hasegawa A, Ohtsubo K, Mori W: Pineal gland in old age; quantitative and qualitative morphological study of $168 \mathrm{hu}-$ man autopsy cases. Brain Res 409:343-349, 1987

19. Jinkins JR, Xiong L, Reiter RJ: The midline pineal "eye": MR and CT characteristics of the pineal gland with and without benign cyst formation. J Pineal Res 19:64-71, 1995

20. Kiely MJ: Neuroradiology case of the day. Pineal cyst with cerebral aqueduct obstruction. AJR Am J Roentgenol 160:1338-1339, 1993

21. Kitayama J, Toyoda K, Fujii K, Ibayashi S, Sugimori H, Sadoshima S, et al: [Recurrent aseptic meningitis caused by rupture of a pineal cyst.] No To Shinkei 48:1147-1150, 1996 (Jpn)

22. Klein P, Rubinstein LJ: Benign symptomatic glial cysts of the pineal gland: a report of seven cases and review of the literature. J Neurol Neurosurg Psychiatry 52:991-995, 1989

23. Koenigsberg RA, Faro S, Marino R, Turz A, Goldman W: Imaging of pineal apoplexy. Clin Imaging 20:91-94, 1996

24. Leone M, D’Amico D, Moschiano F, Fraschini F, Bussone $\mathrm{G}$ : Melatonin versus placebo in the prophylaxis of cluster headache: a double-blind pilot study with parallel groups. Cephalalgia 16:494-496, 1996

25. Mamourian A, Towfighi J: MR of pineal cysts. AJNR Am J Neuroradiol 15:1796-1797, 1994

26. Mandera M, Marcol W, Bierzyńska-Macyszyn G, Kluczewska E: Pineal cysts in childhood. Childs Nerv Syst 19:750-755, 2003

27. Masruha MR, de Souza Vieira DS, Minett TS, Cipolla-Neto J, Zukerman E, Vilanova LC, et al: Low urinary 6-sulpha- 
toxymelatonin concentrations in acute migraine. J Headache Pain 9:221-224, 2008

28. Mena H, Armonda RA, Ribas JL, Ondra SL, Rushing EJ: Nonneoplastic pineal cysts: a clinicopathologic study of twenty-one cases. Ann Diagn Pathol 1:11-18, 1997

29. Michielsen G, Benoit Y, Baert E, Meire F, Caemaert J: Symptomatic pineal cysts: clinical manifestations and management. Acta Neurochir (Wien) 144:233-242, 2002

30. Milroy CM, Smith CL: Sudden death due to a glial cyst of the pineal gland. J Clin Pathol 49:267-269, 1996

31. Morgan JT, Scumpia AJ, Webster TM, Mittler MA, Edelman M, Schneider SJ: Resting tremor secondary to a pineal cyst: case report and review of the literature. Pediatr Neurosurg 44:234-238, 2008

32. Müller-Forell W, Schroth G, Egan PJ: MR imaging in tumors of the pineal region. Neuroradiology 30:224-231, 1988

33. Musolino A, Cambria S, Rizzo G, Cambria M: Symptomatic cysts of the pineal gland: stereotactic diagnosis and treatment of two cases and review of the literature. Neurosurgery 32:315-321, 1993

34. Nolte I, Brockmann MA, Gerigk L, Groden C, Scharf J: TrueFISP imaging of the pineal gland: more cysts and more abnormalities. Clin Neurol Neurosurg 112:204-208, 2010

35. Peres MF: Melatonin, the pineal gland and their implications for headache disorders. Cephalalgia 25:403-411, 2005

36. Peres MF, Zukerman E, Porto PP, Brandt RA: Headaches and pineal cyst: a (more than) coincidental relationship? Headache 44:929-930, 2004

37. Pu Y, Mahankali S, Hou J, Li J, Lancaster JL, Gao JH, et al: High prevalence of pineal cysts in healthy adults demonstrated by high-resolution, noncontrast brain MR imaging. AJNR Am J Neuroradiol 28:1706-1709, 2007

38. Richardson JK, Hirsch CS: Sudden, unexpected death due to "pineal apoplexy". Am J Forensic Med Pathol 7:64-68, 1986

39. Sawamura Y, Ikeda J, Ozawa M, Minoshima Y, Saito H, Abe $\mathrm{H}$ : Magnetic resonance images reveal a high incidence of asymptomatic pineal cysts in young women. Neurosurgery 37:11-16, 1995

40. Seifert CL, Woeller A, Valet M, Zimmer C, Berthele A, Tölle T, et al: Headaches and pineal cyst: a case-control study. Headache 48:448-452, 2008

41. Steven DA, McGinn GJ, McClarty BM: A choroid plexus papilloma arising from an incidental pineal cyst. AJNR Am J Neuroradiol 17:939-942, 1996

42. Sun B, Wang D, Tang Y, Fan L, Lin X, Yu T, et al: The pineal volume: a three-dimensional volumetric study in healthy young adults using 3.0 T MR data. Int J Dev Neurosci 27:655-660, 2009

43. Tajima Y, Minami N, Sudo K, Moriwaka F, Tashiro K: Hot water epilepsy with pineal cyst and cavum septi pellucidi. Jpn J Psychiatry Neurol 47:111-114, 1993

44. Tamaki N, Shirataki K, Lin TK, Masumura M, Katayama S, Matsumoto S: Cysts of the pineal gland. A new clinical entity to be distinguished from tumors of the pineal region. Childs Nerv Syst 5:172-176, 1989

45. Tanuri FC, de Lima E, Peres MF, Cabral FR, da Graça Naffah-Mazzacoratti M, Cavalheiro EA, et al: Melatonin treatment decreases c-fos expression in a headache model induced by capsaicin. J Headache Pain 10:105-110, 2009

46. Tapp E: The histology and pathology of the human pineal gland. Prog Brain Res 52:481-500, 1979

47. Tien RD, Barkovich AJ, Edwards MS: MR imaging of pineal tumors. AJR Am J Roentgenol 155:143-151, 1990

48. Wisoff JH, Epstein F: Surgical management of symptomatic pineal cysts. J Neurosurg 77:896-900, 1992

\section{Disclosures}

The authors report no conflict of interest concerning the materials or methods used in this study or the findings specified in this paper.

\section{Author Contributions}

Conception and design: Sherman, Starke. Acquisition of data: Sherman, Starke. Analysis and interpretation of data: Starke, Cappuzzo. Drafting the article: Starke, Cappuzzo, Erickson. Critically revising the article: all authors. Reviewed submitted version of manuscript: all authors. Approved the final version of the manuscript on behalf of all authors: Sherman. Statistical analysis: Starke. Study supervision: Sherman.

\section{Supplemental Information Current Affiliations}

Dr. Starke: Department of Neurosurgery and Radiology, University of Miami, Miami, FL.

\section{Correspondence}

Jonathan H. Sherman, Department of Neurosurgery, The George Washington University, 2150 Pennsylvania Ave. NW, Ste. 7-420, Washington, DC 20037. email: jsherman0620@gmail.com. 